\title{
Introduction to Wavelets and their applications in signal denoising
}

\author{
Çiğdem Polat Dautov a,* , Mehmet Siraç Özerdem ${ }^{\mathrm{b}}$ \\ a Bitlis Eren University, Department of Electrical and Electronics Engineering, TR-13000, Bitlis Turkey \\ b Dicle University, Department of Electrical and Electronics Engineering, TR-21280, Diyarbakır Turkey
}

\section{ART I C LE INFO}

Article history:

Received 03 November 2017

Received in revised form 16 February 2018

Accepted 07 March 2018

\section{Keywords:}

Fourier transform

Signal denoising

Short time Fourier transform

Wavelet transform

\begin{abstract}
A B S T R ACT
The aim of this study is providing a comprehensive background information related to the roots of both Fourier Transform (FT) and Wavelet Transform (WT) along with an experiment related to applications of WT techniques. The paper describes several applications of WT and provides background information on FT. Fourier Transform (FT) is a concept that has a long history yet several issues related to resolution and uncertainty of time -frequency. Even though there are several adapted forms of FT such as Short Time Fourier Transform (STFT), which intend to solve the problems, certain limitations remain. Wavelet Transform (WT) is an alternative transformation technique emerged in order to fully tackle these diverse and complicated issues. In this paper, the background information related to the roots of FT and WT are given. Some of the problems that WT addresses are examined. WT is a tool that has many advantages among them is noise reduction and compression. We reviewed several studies that use the noise reduction capability of WT alone or combined with other signal processing tools. Discrete Wavelet Transform (DWT) based algorithm is also examined as a noise reduction technique and carried out in MATLAB setting. Analysis on a speech signal which contaminated with keyboard sound also a number spelling female voice containing unknown noise are performed. Different types of thresholding and mother wavelets were in consideration and it was revealed that Daubechies family along with the soft thresholding technique suited our application the most.
\end{abstract}

(C) 2017. Turkish Journal Park Academic. All rights reserved.

\section{Introduction}

Even though wavelets have been around for a relatively short period, it is worth mentioning what led researches to their invention. Thus, a very well-known and understood concept, FT is introduced. Transformation (transform) is a mathematical operation defined as a change of basis functions. In vector space transform is a linear operation (linear mapping) that expresses any vector as a set of weighted linearly independent vectors. Mathematical transformations can be applied to many fields like signal processing aimed at obtaining information by transforming raw signal into a processed signal. Any signal in time domain can be defined as raw signal that has not been transformed by any mathematical transformation. On the other hand, a processed signal is a raw signal that has been transformed to a different format where it *Corresponding author

Tel.: +90 4342220000; Fax: +904342220031

E-mail address: cigdembilmuh@hotmail.com is represented by a new set of functions (Polikar, 1994). Raw signal can only give the amplitude change over time whereas

processed signal can reveal more information about the behaviour of the signal in different domains, i.e. frequency domain. Since in practice, most signals exist in time domain where they are represented in time-amplitude format, it is not easy to extract valuable information, and transforming these signals becomes a necessity. There are several common mathematical transformations which are used in signal processing such as Fourier Transform, Short Time Fourier Transform, and Hilbert Transform, Wigner distributions, Wavelets, the Radon transform... etc. Each one of them comes with different advantages and disadvantages. The aim of this study is describing some of the most important transformation 
methods, providing background information related and giving several real life applications in the field of signal processing.

FT was one of the first that emerged in early 19th century as a mathematical approach which suggests representation of any signal as an infinite sum of sine and cosine waves (Osgood, 2007). The most significant reason why FT has been used in the field of signal processing is its simple yet efficient way of representing a signal in frequency domain while being able to convert it back to time (Osgood, 2007). Types of FT can be applied to periodic, aperiodic and continuous or discrete signals (Bouman, 2013). The Fourier Transform is an extended version of Fourier series where the original signal is represented as the collection of peak values of the signal, which is converted to sine and cosine waves in frequency domain.

Fourier transform is a continuous process letting the converted signal last infinite in theory. It is mathematical representation is given below:

$X(j \omega)=1 \int_{-\infty}^{+\infty} x(t) e^{-j \omega t} d t$

$x(t)=\frac{1}{2 \pi} \int_{-\infty}^{+\infty} X(j \omega) e^{j \omega t} d \omega$

The first equation is the mathematical representation of FT, which shows that is possible to represent a function in time (original signal) as a function in frequency (decomposed signal). Second equation, on the other hand, shows that reversing Fourier Transform makes it possible to compose the original function back in time domain without any lost (Huang \& Macfarlane, 2012).

As the mathematical aspect of the matter are covered, the benefits of using FT in signal processing applications will be discussed. For example, the shape of a biological signal such as Electrocardiography (ECG) is generally well known to doctors. However, it is not always easy to see small deviations occurring within the signal (Yadav, 2016). ECG signals are naturally available in time-domain that makes it difficult for cardiologists to detect pathological conditions. With the help of FT new ECG measuring equipment allow doctors to see signal in both time and frequency domain. It facilitates diagnoses of heart conditions.

FT essentially allows engineers to observe sudden changes in the signal by altering the domain of signal from time to frequency. This way the unexpected changes can be identified, interpreted and analyzed. FT is currently in use for analyzing the natural signals, transferred data in a network or for detecting and filtering signals in case of interference and/or distortion. FT has different forms that are dedicated to solve certain problems. Continuous Fourier Transform, Discrete Fourier Transform, Fast Fourier Transforms can be considered as the most important type of FT techniques (Huang \& Macfarlane, 2012).

Theoretically, FT is an infinite process that proposes taking a signal existing in continuous time and transforming it into frequency domain. Continuous Fourier transform fundamentally suggests an infinite approach for representation of a signal lengthened and allowed to approach infinity. Even if it is possible continuously representing a signal, using Fourier series, in real world applications since sources are limited, the FT should be rather finite. Discrete Time Fourier Transform (DTFT) can allow this under certain theoretical conditions described as the sampling theorem that suggests the possibility of perfect recovery of original continuous function from DTFT and thereby from the original discrete samples. The algebraic representation of DTFT is given below:

$\mathrm{X}(\mathrm{k})=\sum_{n=0}^{N-1} x(n) e^{-j w n k}$

$\mathrm{x}(\mathrm{n})=\frac{1}{N} \sum_{k=0}^{N-1} X(k) e^{j w n k}$

, where $w=\frac{2 \pi}{N}$ and $\mathrm{k}=0$ to $\mathrm{N}-1$.

As it is shown, DTFT's mathematical expression is very similar to the original FT's (Eq.1 to Eq 3 and Eq.2 to Eq 4). DTFT itself is actually a continuous function of frequency but the discrete samples calculated via discrete Fourier transform can make the whole transform discrete time.

Despite the efficiency of FT, there are certain situations where it may not provide the best performance. FT works well, when it is applied to stationary signals. However, it doesn 't give the desired outcome when it is applied to nonstationary signals (Hazas \& Hall, 1999). The concepts of stationary and nonstationary signals define the properties of the process that generates the signal. Signals whose frequency content do not change in time are called stationary signals whereas signals whose frequency content varies in time are called nonstationary (Polikar, 1994). Take white noise as an example of stationary signals, any signal value taken in different time instances are equally probable to happen. A commonly given example, chirp sound is on the other hand a non-stationary process because its frequency components change over time. To have a better understanding, we compared the FT results of both stationary and non-stationary signals whose frequency components are similar. The stationary signal (Figure 1a) is represented as:

$$
\begin{aligned}
\mathrm{x} 1(\mathrm{t})=\cos (2 * \mathrm{pi} * \mathrm{f} 1 * \mathrm{t})+\cos (2 * \mathrm{pi} * \mathrm{f} 2 * \mathrm{t}) \\
+\cos (2 * \mathrm{pi} * \mathrm{f} 3 * \mathrm{t})+\cos (2 * \mathrm{pi} * \mathrm{f} 4 * \mathrm{t})
\end{aligned}
$$

Where $\mathrm{f} 1, \mathrm{f} 2, \mathrm{f} 3, \mathrm{f} 4$ are given as $5,10,25,50 \mathrm{~Hz}$ respectively. The non-stationary signal (Figure 1b) also contains same frequency components but in different time instances. It can be represented as:

$$
\begin{aligned}
\mathrm{x} 1(\mathrm{t})=\cos (2 * \mathrm{pi} * \mathrm{f} 1 * \mathrm{t} 1)+\cos (2 * \mathrm{pi} * \mathrm{f} 2 * \mathrm{t} 2) \\
+\cos (2 * \mathrm{pi} * \mathrm{f} 3 * \mathrm{t} 3)+\cos (2 * \mathrm{pi} * \mathrm{f} 4 * \mathrm{t} 4)
\end{aligned}
$$



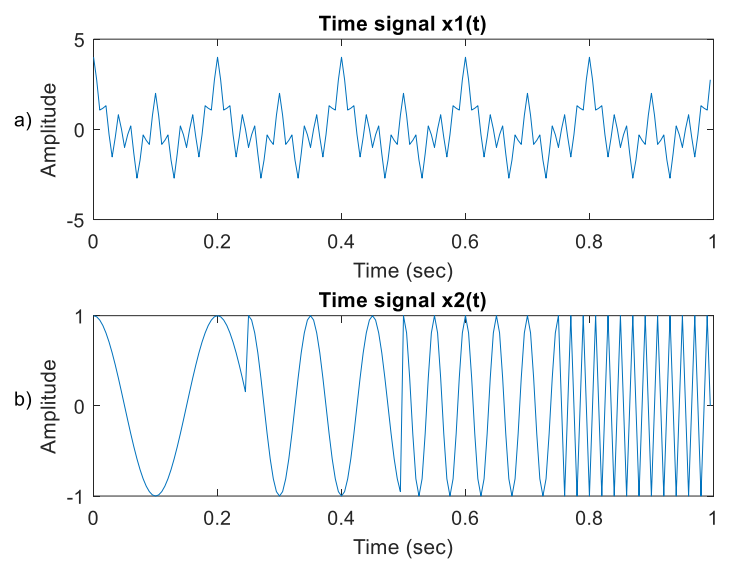

Figure 1. Signals $\mathrm{x} 1$ and $\mathrm{x} 2$ in time domain

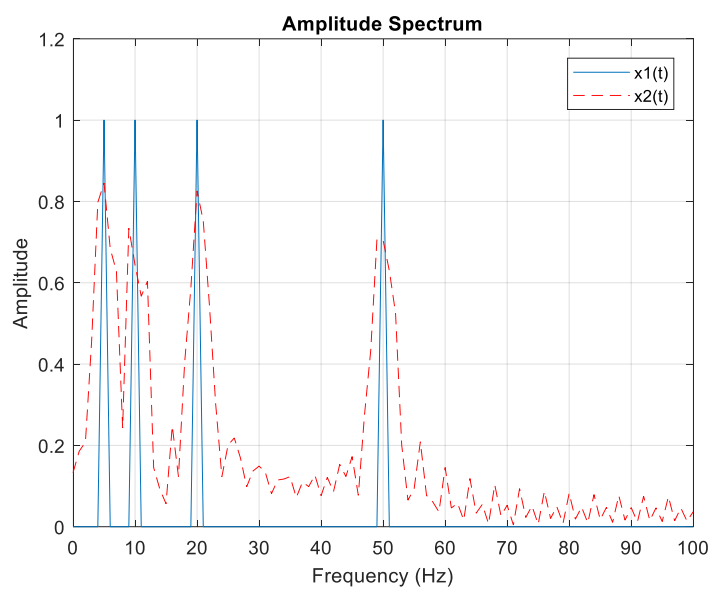

Figure 2. Signals $\mathrm{x} 1$ and $\mathrm{x} 2$ in frequency domain

After applying FT to both signals, the frequency domain representations of both signals given in Figure 2. As you can see in the figure, we observe similar frequency spectrums although the corresponding time-domain signals are not the same. This happens since FT is a technique that does not provide both frequency and time information simultaneously. When the signal is stationary it works perfectly fine but when the signal is randomly changing, crucial information on when certain frequencies appear in time gets lost.

To tackle the aforementioned issue a modified FT technique named Short Time Fourier Transform (STFT) was proposed. STFT is a process that can be defined as FT of the original signal multiplied by a window function (Federico \& Kaufmann, 2009). This function allows us to divide the non-stationary signal into smaller segments, which have higher chance being stationary. It is mathematical representation is given below:

$\operatorname{STFT~} x(\omega)\left(t^{\prime}, f\right)=\int_{1}\left[x(t) * \omega^{*}\left(t-t^{\prime}\right)\right] * e^{-j 2 \pi f t} d t$

By giving different values to $t^{\prime}$, we are changing the width of the window function allowing us to examine longer or shorter portions of the signal. There is a correlation between the width of a window and the certainty of frequency. Wider window provides better frequency resolution. In this case, we compromise, the certainty of time. When the window is narrower, the fewer frequency components fall into it compromising frequency resolution (Liu, 2010). These phenomena root back to the Heisenberg Uncertainty Principle. The Heisenberg Uncertainty Principle states that it is impossible to know the exact time-frequency information (called as time-frequency resolution) (Figure3). In order to have better time-frequency resolution, Wavelet emerged later in 1960's allowing to represent signal both in time and frequency domain simultaneously. This way, not only both time and frequency information is available, it is also possible to store signal efficiently.

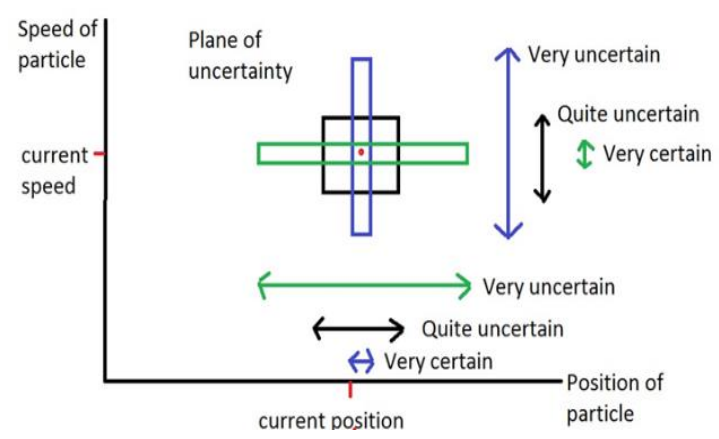

Figure 3. Plane of uncertainty (Liu, 2010)

Mathematically, Wavelets are nothing but functions that divide the original signal into different frequency components and study each component. The basis functions of Wavelet Transform (WT) are scaled according to the frequency. There are different small waves (also known as mother wavelets) that can be used for the implementation of WT (Cengiz \& Arıöz, 2016). The mother wavelet is actually a window function that moves forward in time. Unlike STFT, it is possible to use different types of window functions by using these mother wavelets and performing the decomposition of the signal $\mathrm{x}(\mathrm{t})$ into weighted set of scaled wavelet functions $y(t)$.

Dubachies, Haar, Symlet, Coiflet, Mexican Hat, Morlet wavelets can be given as some of the different types of wavelets (Figure 4). Each of them comes from different wavelet families and includes different properties (Cengiz et al., 2016). When we are applying these wavelets, we decide which one to use depending on the requirements of the application. 

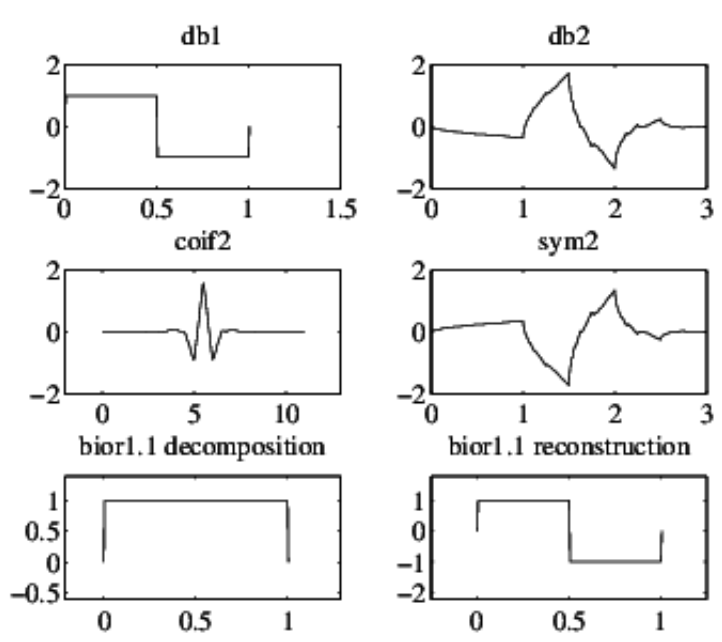

Figure 4. Some of the Wavelet families.

The main idea behind the WT is decomposing a signal into different frequency level of coefficients. Fundamental capability of analyzing signal in different frequency bands and scales can be helpful determining signals behaviors' in different sections of frequency. Thus, detecting the corruption within the signal and eliminating it becomes a relatively easy process.

There are two types of WT defined as Continues Wavelet Transform (CWT) and Discrete Wavelet Transform (DWT). CWT's mathematical representation is given as:

$\operatorname{CWT}(\mathrm{a}, \mathrm{b} ; \mathrm{x}(\mathrm{t}), \psi(\mathrm{t}))=\int_{-\infty}^{\infty}\left[\mathrm{x}(\mathrm{t}) \frac{1}{\mathrm{a}} \psi^{*}\left(\frac{\mathrm{t}-\mathrm{b}}{\mathrm{a}}\right)\right] \mathrm{dt}$

, where $x(t)$ is the original signal, $\psi(t)$ is the analysing function (wavelet), $\mathrm{a}$ is a scale parameter and $\mathrm{b}$ is a position in time.

CWT uses inner products of analyzing function and the original CWT signal and measures the similarity between these two functions by integration (Federico \& Kaufmann, 2009). Changing scale parameter and position parameter, CWT allows us to shift and compress or stretch the mother wavelet in order to capture multiple frequency components at different locations (Figure 5).

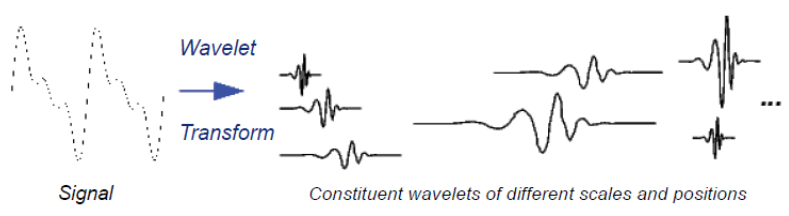

Figure 5. Scaling and positioning by CWT.

Considering $\psi(t)$ as the bandpass impulse response, scaling the wavelet varies the bandwidth of the bandpass. Contrary to STFT, CWT allows changing the support of the wavelet to get better resolution in frequency domain. In Figure 6, we can observe the resolution difference between CWT and STFT. As we see there are overlaps in the magnitude scalogram of STFT while there is a clear separation between frequency components that exist in different time intervals (Misiti, Misiti, Oppenheim, \& Poggi, 2009).
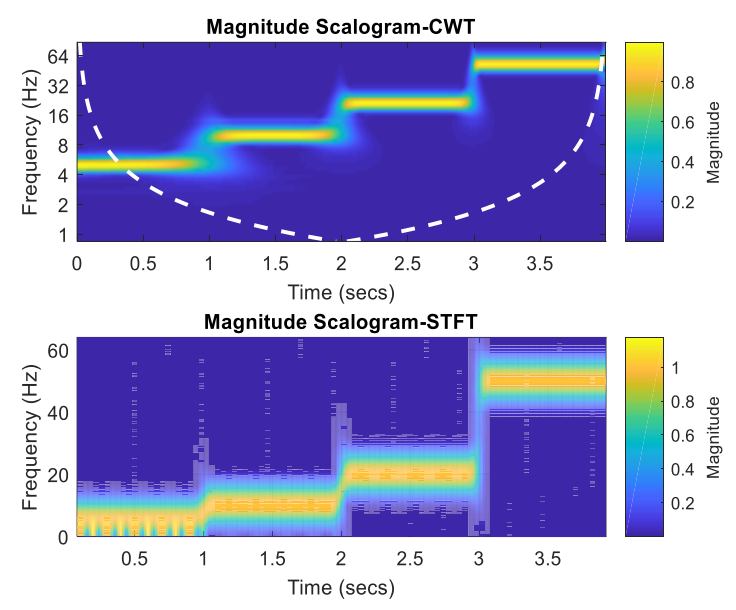

Figure 6. Magnitude scalograms of CWT and STFT.

As it is mentioned previously, another type of WT exists in literature called DWT which is an implementation of WT using mutually orthogonal set of wavelets defined by carefully chosen scaling and translation parameters ( $a$ and b). This leads to a very simple and efficient iterative scheme for doing the transformation (Misiti et al., 2009). The scaling function should meet several requirements such as orthogonality, the normalized area between the functions etc... The translation equation is given as:

DWT $\left[n, a^{\mathrm{j}}\right]=\sum_{m=0}^{\mathrm{N}-1} \mathrm{x}[\mathrm{m}] . \psi_{\mathrm{j}}^{*}[\mathrm{~m}-\mathrm{n}], \quad \psi_{\mathrm{j}}[\mathrm{n}]=\frac{1}{\sqrt{\mathrm{a}^{\mathrm{j}}}} \psi\left(\frac{\mathrm{n}}{\mathrm{a}^{\mathrm{j}}}\right)$

where $\mathrm{n}$ is delay parameter, $\mathrm{N}$ is the length of signal, $\psi$ is the discretized mother wavelet.

Since in DWT, computation performed on discrete set of wavelets, it provides a significant yield in terms of computational time. Therefore, DWT is useful when compression of the signal is needed. However, this property comes at a price, especially when the main purpose is to analyze data to discover hidden information.

\section{The applications of WT}

WT is a powerful tool among researchers in different fields. WT is implemented in various fields in order to accomplish several tasks such as data and image compression, partial differential equation solving, transient detection, texture analysis, noise/trend reduction. In next section, several case studies related to the de-noising applications of WT will be examined and in the end of the case studies, the implementations of denoising algorithm to eliminate common office environment noises will be presented. Before giving the case studies, it should be noted that DWT stands for Discrete Wavelet Transform while IDWT stands for inverse DWT that is the process of reconstructing the original signal. 


\subsection{Case Study I}

Electrodes attached to the surface of the scalp can measure electroencephalogram (EEG) signals that are electrical impulses produced by nerves in the brain. EEG signals are useful for doing deductions about the mental state of a person and diagnosing a number of diseases such as epilepsy, brain tumors, and trauma caused problems. EEG signal is random and non-stationary signal that is very weak to identify. Due to its nature, it is also almost impossible to capture EEG signals without any introduction of unwanted signals generated by body, electrode or power line movement. Considering how delicate the health matters are, appropriate analysis of EEG signals can be only possible by the reduction of noise within the signal before any further analysis (S. S. Patil \& Pawar, 2012). De-noising EEG data is a quite complicated process due to variety of possible noise involved in the process of capturing the EEG signal. Another matter with EEG signal recording and analysing is the weak EEG signals (1-100 Hz) brings the requirement of amplification of the EEG signals in order to do any synthesis. The amplification of EEG signals also is the source of amplified noise, which requires an efficient signal deduction method.

In reference study (S. S. Patil \& Pawar, 2012), a method for denoising of the EEG signal is introduced by applying discrete wavelet transform. As a consequence of the continues nature of the CWT, it is not possible to process a real signal on a digital computer that is why another form of wavelets named DWT is used.

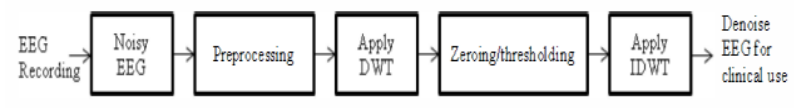

Figure 7. The diagram of the proposed method (reference study).

The de-noising process generally involves three steps which are given as decomposition of the signal at a certain level, zeroing or thresholding the detail coefficients, reconstructing the signal using the original approximation coefficients and modified detail coefficients at certain level in order to verify the outcome (Figure 7 and 8).

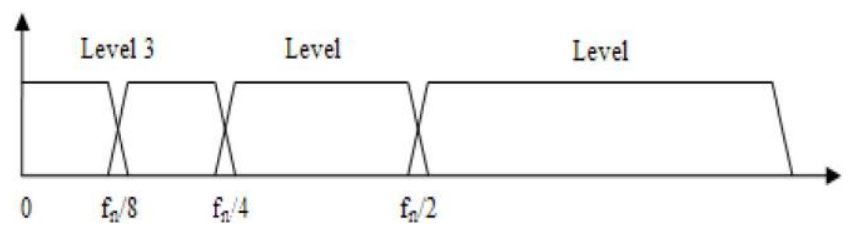

Figure 8. Frequency domain representation of decomposed signal (reference study).

In order to illustrate how the Wavelet de-noising works, the researchers of the study used the EEG database available that is referred within the study. Before the wavelet de-noising start, the EEG signals were amplified. After this stage, there were several filtering applications completed meant for removing low and high frequency interference. Five different types of wavelets named as Haar, Daubechies, Symlets, Coiflets and Dmey were applied to the data set; the performance of each wavelet was evaluated. The evaluation criteria were defined as the signal noise ratio (SNR) and mean square error (MSE). The results of this study, shows that hard thresholding produces better results than soft thresholding in terms of noise reduction. It is also seen that global threshold works much better than local threshold. Removing all the details of the original signal and moving forward with the approximation coefficients has an outcome of increasing the efficiency of denoising process. The reader is referred to 'Wavelet Transform to Advance the Quality of EEG Signals in Biomedical Analysis' for more information (S. S. Patil \& Pawar, 2012)

\subsection{Case Study II}

Electrocardiography (ECG) is a technique using electrodes attached on the skin in order to record the electrical activities of the heart over an episode of time. ECG is in use for medical purposes such as monitoring the health of heart, detecting any usual activity that can indicate cardiac diseases, monitoring the effects of a medicine (Guo, Li, Suo, \& Liang, 2017). However, ECG signals may be corrupted by baseline wander or some kinds of high frequency noise. It is also possible have multiple distortions on an ECG signal cause by different noise sources. It is known that removing noise from an ECG signal is a complicated process that can cause severe R-wave attenuation and thus result in wrong diagnoses.

In reference study (Guo et al., 2017); a new technique proposed that is capable of removing mixed noises using Discrete Wavelet Transform (DWT) in unconventional approach where separate noise estimators calculated by the use of multilevel DWT (Figure 9). What is new about the technique is a set of detail and approximation coefficients is used to do estimations for different types of noise. In fact, DWT gives two singular coefficient sets as approximation and detailed coefficients. This method uses approximation coefficients in order to estimate low frequency noise where detailed coefficients used to estimate high frequency noise by reconstructing waveforms out of the coefficients. Conventionally the DWT method eliminates the noise with adaptive thresholding. Although this method provides good performance, it requires great amount of computations. The new approach on the other hand, achieves the same performance as the conventional DWT denoising technique but spares us from the high computational complexity (Figure 10).

For the simulation section of the study, MIT-BIH arrhythmia database was used. The ECG was corrupted with a simulated baseline wander and a Gaussian noise ( $\mu=0$ and $\sigma=0.0625)$. In the part of the simulation where the new method was introduced, the approximation coefficients were used to estimate the baseline wander and the threshold applied detail coefficients to estimate high frequency noise. In order to have a good comparison, the conventional DWT and another method called empirical mode decomposition (EMD) were applied to the noisy signal. As a wavelet form Daubechies 4 was used and in the case of EMD the first three and last four instruct mode functions were subtracting from the noisy ECG. For the case of both DWT, the first five levels of detail coefficients were thresholded. 


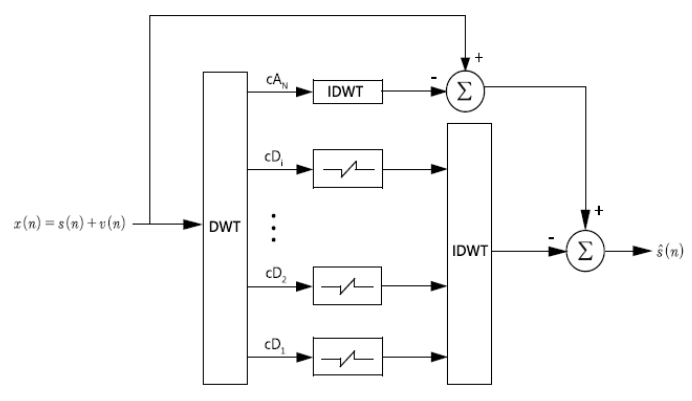

Figure 9. The proposed structure based on the reference study.

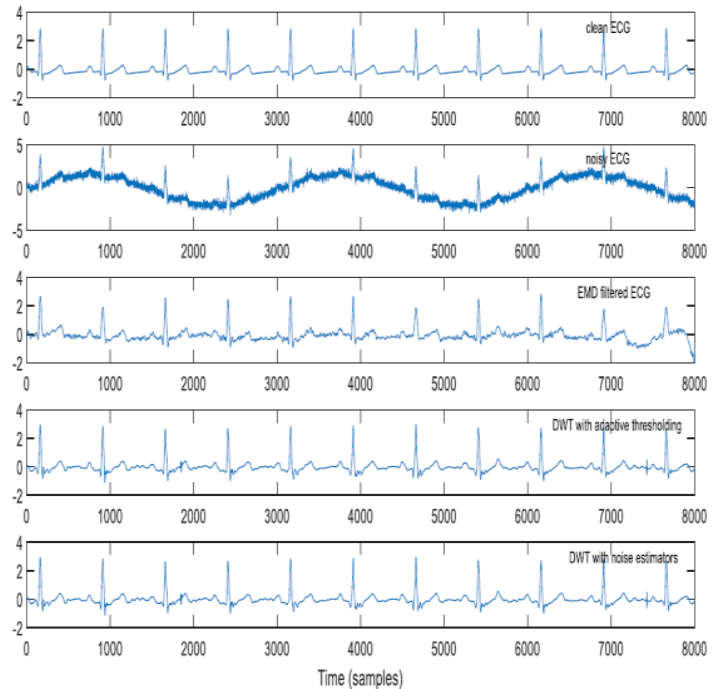

Figure 10. The comparison of different approaches applied to noisy ECG (reference study).

Based on the results of experiment, it can be said that EMD performs poorer than the other two methods where the new method performs the same as the method using adaptive thresholding technique (Aggarwal et al., 2011). However, a significant reduction of computation complexity is possible with the new approach compared to the other two methods using adaptive thresholding techniques (Figure 10). The performance of the new approach is significantly good in terms of both de-noising signal and lowering the process complexity.

\subsection{Case Study III}

Detecting any signal that is not artificial, without noise is almost impossible to do so. Researchers working the field of signal processing very often may encounter the problem of eliminating the noise within the signal. This fact results different attempts and trials in order to find the best method to do noise reduction. There are several methods used for denoising such as Fourier Transform (FT), Wavelet Transform (WT) and Singular Vector Decomposition (SVD). FT is a method that gives a beneficial result only when it is applied to stationary signals where WT, SVD are the methods that give a good result both for stationary and non-stationary signals. In this study, both WT and SVD are used to create a combined new method for eliminating noise(R. Patil, 2015).

Studies that aim to target the noise mixed into signal and eliminate it, generally solves the problem in two fundamental ways. The first one is de-noising signal while it is in the original signal domain where FT is generally the transformation method for it; the second way is de-noising signal while it is transformed into another domain where WT is the suitable method to do the transformation (R. Patil, 2015). For many fields like signal, image processing and pattern recognition, WT is a powerful tool, which gives reliable and detailed time-scale information of the signal helping to eliminate the noise. SVD is also another mathematical method that can identify and order the dimensions along the data points exhibit the most variations. By applied the SDV to a signal, it is possible to find the best approximation of the original signal with less dimensions. So that applying SVD can reduce the noise, also compress the signal itself. The reference study [3] introduces a new method where SVD and WT are used together in order to reduce the noise. Matlab was used as an experimental setup and an artificial signal (Figure 11) was created as:

$$
\begin{gathered}
x(t)=0.1 * \sin \left(2 * \pi * 100 *\left(\frac{\mathrm{t} 1}{1000}\right)\right)+\sin \left(2 * \pi * 50 *\left(\frac{\mathrm{t} 2}{1000}\right)\right) \\
+\sin \left(2 * \pi * 25 *\left(\frac{\mathrm{t} 3}{1000}\right)\right)+0.1 * \sin \left(2 * \pi * 10 *\left(\frac{\mathrm{t} 4}{1000}\right)\right)
\end{gathered}
$$

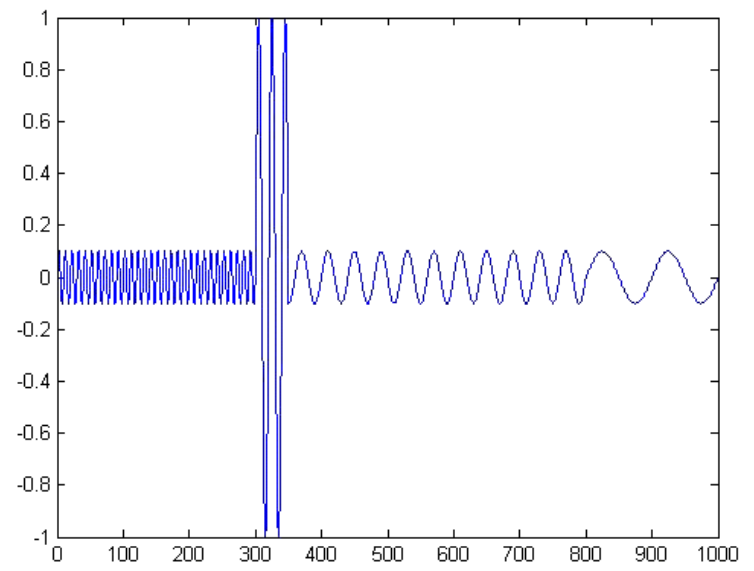

Figure 11. Artificially created signal $x(t)$ (reference study)

Later on Gaussian (White) noise was introduced to the signal in the ratio of 1,5 and $10 \mathrm{db}$. Morelet wavelet was picked for the wavelet transform. In an order, coefficients of the original signal targeted to be de-noised were transformed using Morelet wavelets and later coefficients were further processed using SVD. The process was repeated 100 times in order to see the difference between the original signal and noisy signal processed with only WT and also between original signal and processed noisy signal with both SVD and WT. The results of this study show that applying both SVD and WT eliminates the signal better compared to only WT applied one. SNR which can be interpreted as a success rate of the method shows 4-5 times higher SNR when SVD and WT applied together (R. Patil, 2015). 


\section{Wavelet denoising experiment}

In order to illustrate how the Wavelet de-noising works, we conducted two different experiments. For the first experiment, a male speech using the given different forms of audio samples were analyzed: desired (original) signal, signal containing keyboard sound as a noise and noise sound itself. For the second experiment, a sample female sound was investigated. For this experiment, only noisy signal form was available and no other information. For the experiments the sampling frequency is defined as Fs $=1600 \mathrm{~Hz}$. DWT method is used in order to eliminate the noise with adaptive thresholding. By running the algorithm steps iteratively, we established the ideal threshold technique, type and mother wavelets for each experimental samples. It should be noted that even though this method provides good performance, it requires great amount of computations.

\subsection{Algorithm}

The algorithm that is used for the purpose of de-noising our sample signals consist of three different fundamental iterations (Yadav, 2016). The scheme of the Wavelet denoising algorithm is given in the Figure 11.

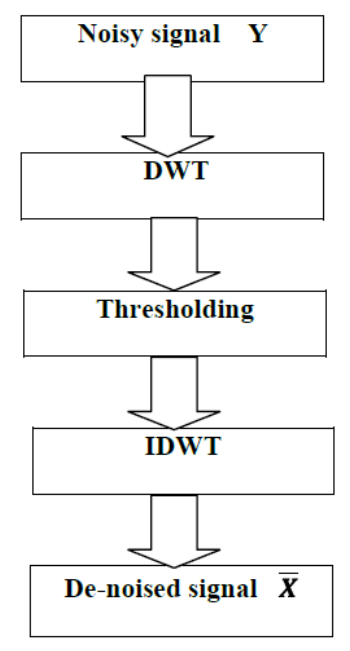

Figure 12. The algorithm steps.

In order to de-noise any signal, we need to put the noisy signal into the decomposition process by applying wavelet transform. Wavelet transform allows us to decompose signal into groups of coefficients at different frequency levels. After successfully applying the first stage, it is possible to obtain relevant information related to the signal characteristic. Understanding how signal behaves in different frequency segments will allow us to select the most suitable threshold in the next stage. The next step is determining the best threshold values and applying threshold values to these set of coefficients so it is possible to eliminate unwanted data. The thresholding technique will be covered in the next section. Final step of the algorithm is where we use these filtered coefficient sets to recompose our signal.
For this to be possible an inverse discrete Wavelet transform is done.

\subsection{Thresholding}

The most important thing in applications of thresholding is the determination of threshold value. There are four different estimator of threshold value given as heasure, minimax, rigsure and sqtwolog. Minimax and SURE threshold selection rules are more convenient when small details of the signal lie near the noise range (Aggarwal et al., 2011). For the other cases the rest two give more efficient outcomes.

Thresholding can be also applied in two different methods: hard and soft thresholding. Hard threshold can be explained as setting elements to zero where their absolute values are lower than the threshold (Eq. 11). Hard threshold gives sharper results. On the other hand, soft thresholding softens the coefficients exceeding the threshold by lowering them as much as threshold value (Eq. 12). Soft thresholding requires more computations but gives better denoising performance (Aggarwal et al., 2011). An example is given in Figure 13 about how hard and soft thresholding works on a line space $Z=(-1$ 1,100) signal sample.
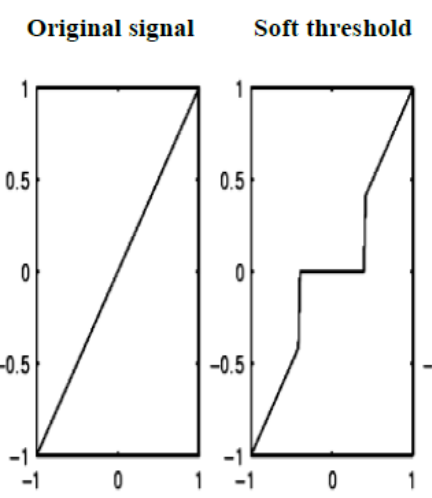

Hard threshold

Figure 13. Soft and hard thresholding.

$$
\mathrm{T}_{\text {hard }}= \begin{cases}x & |x| \geq t h r \\ 0 & |x|<t h r\end{cases}
$$

$$
\mathrm{T}_{\mathrm{soft}}=\left\{\begin{array}{rr}
\operatorname{Sign}(x) \cdot(x-t h r) & x \geq t h r \\
0 \quad-t h r \leq & x<t h r \\
\operatorname{Sign}(x) \cdot(x+t h r) & x<-t h r
\end{array}\right.
$$

\section{Matlab experiments}

\subsection{Experiment I: A male subject typing on the keyboard and reading}

In the first experiment, a sample male speech record where the subject reads and types on the computer keyboard is used. The 
desired output of the first analysis and de-noising process is also known as record with no keyboard sound. The keyboard sound itself is determined as the noise. The first thing we want to examine is plotting all the records defined as desired, noisy signal ( $\mathrm{x}$ ) and noise (Figure 14 and 15).
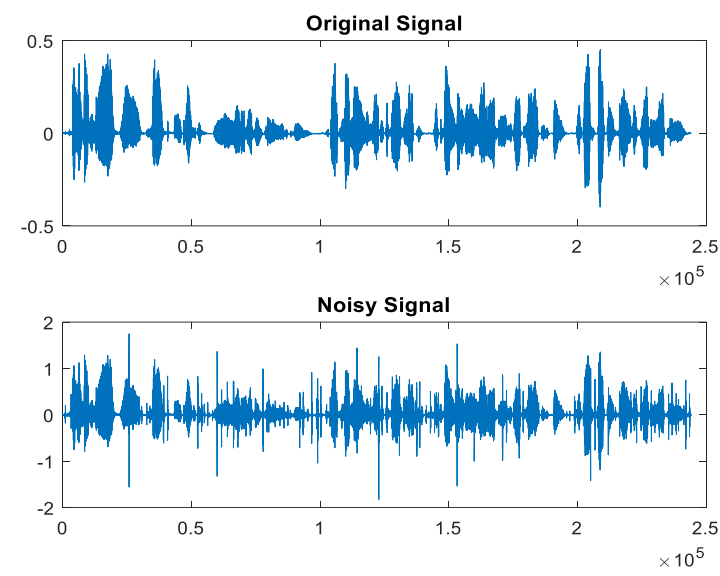

Figure 14. Original and noisy signals in time domain.

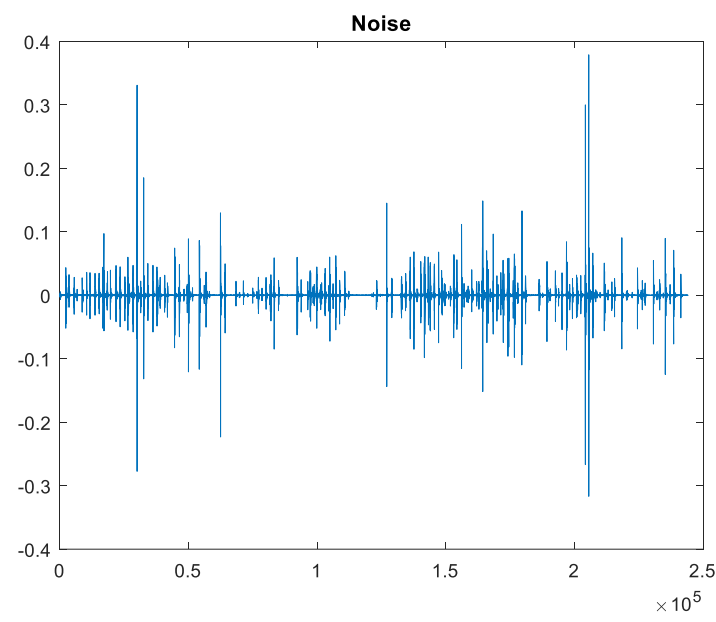

Figure 15. Noise in time domain.

In order to eliminate the noise in the signal the first step is decomposition of signal into approximation and detail coefficients. In this stage even though it was possible to apply one level wavelet transform, a three (level=3) level WT was implemented. As a form of Wavelet, we used db8 since it is one of the most suitable wavelets in this case. As an outcome of the process, the last approximation coefficients and detailed coefficients at level one, two and three were plotted as an illustration (Figure 16).

Next step will be de-noising the signal by using threshold technique. The most important thing we should focus on is selecting the right threshold method and calculation. We move forward with soft thresholding because the signal and noise exist in similar frequency levels and similar amplitudes. For not losing much of an information, soft thresholding is applied.

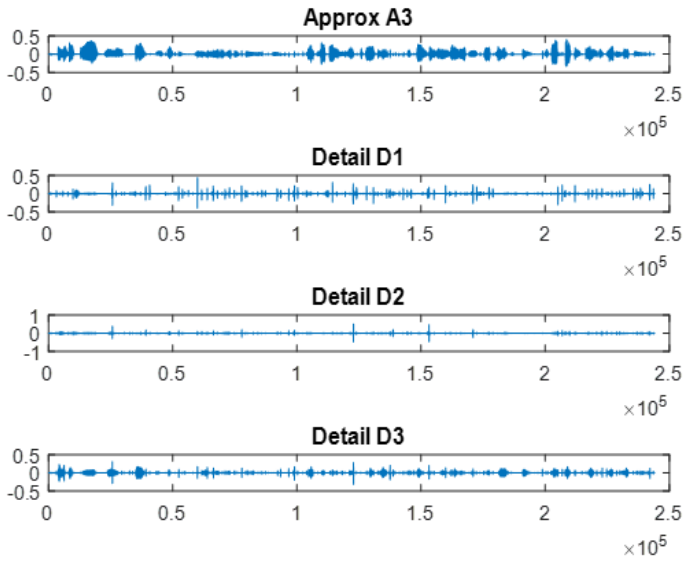

Figure 16. Approximation, detail D1, detail D2, detail D3 coefficients

The threshold calculation method is picked as hearsure for the same reasoning. In order to represent the data after thresholding, we also need to move forward and finish the last stage where we reconstruct our signal as a denoised version of the original signal. In the last step, the results of the de-noising function were represented as original, noisy and de-noised signal given in Figure 17.
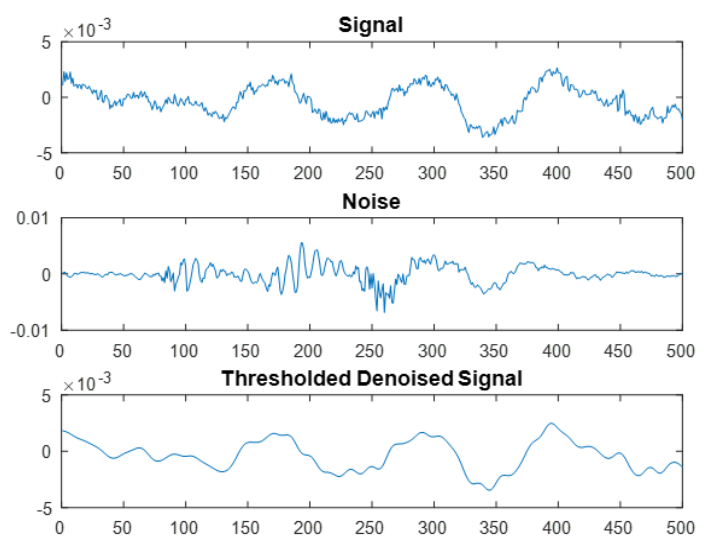

Figure 17. Comparison of original, noise and denoised signals.

\subsection{Experiment II: A female speech with unknown noise}

In the second experiment, a sample female speech record where the subject spells several digits is used. The desired output of the first analysis and de-noising process is unknown. The first thing we want to do is plotting the record, the noisy signal (x) (Figure 18). 


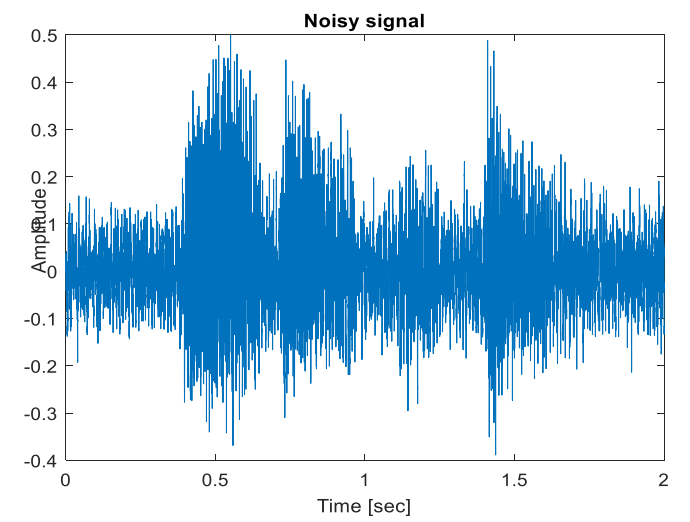

Figure 18. Noisy signal in time domain.

In next step, db4 is chosen as wavelet type since it is one of the most suitable Wavelets. In this experiment different from the previous one, a manual multiple level threshlding was not needed. Since the built-in auto denoising function was enough to calculate and remove the noise from the signal, this function was implemented to denoise the signal at level 5. The threshold technique used by the auto denoising function is defined as modwtsqtwolog. The result of denoising is given in Figure 19.

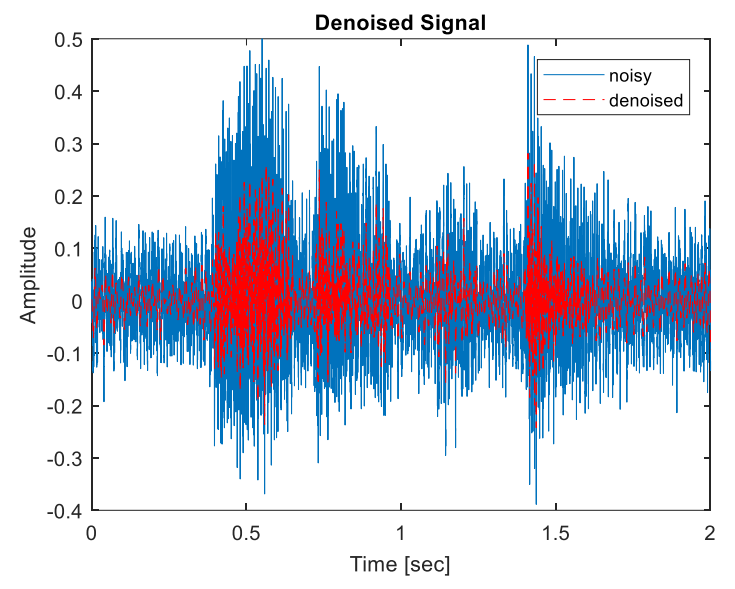

Figure 19. Original signal and noise added versions combined in time domain.

Due to necessity of evaluation of the results, SNR is used as a metric for assessing the outcomes. For that, a prior pre SNR value knowledge was needed. The SNR values gathered after the applications of different wavelets families are given in Table 1. As we can observe, the best SNR outcome is provided by the Daubachies Wavelets.
Table 1. Output SNR values of several wavelets.

\begin{tabular}{lcc}
\hline Wavelet family & Wavelet name & Output SNR \\
\hline Daubechies & $\mathrm{db} 20$ & 13.8179 \\
Daubechies & $\mathrm{db8}$ & 13.8286 \\
Coif & coif2 & 13.7116 \\
Coif & coif5 & 13.7416 \\
Bior & bior3.1 & 11.5034 \\
Bior & bior3.2 & 11.5732 \\
\hline
\end{tabular}

\section{Conclusions}

Within this work, a detailed introduction to Wavelets, continuous and discrete Wavelet Transform was given in a structured manner. An explanation about how they compare to commonly used Fourier Transform and Short-Time Fourier Transform was provided and showed on multiple examples that Wavelet Transform is able to provide better timefrequency resolution. In the next part, we reviewed several research studies where various versions of WT along or in combination with other techniques are used for denoising the signal of interest. This reinforced the fact that noise reduction is one of the most utilized application of WT in multiple different fields of science and engineering. At the end, we carried out our own set of experiments where WT denoising with various underlying wavelet functions and thresholding techniques were applied in two different settings; first with a male subject reading a book out loud and simultaneously typing on a keyboard where keyboard stroke sound was treated as noise. In another one, female was saying digits with additive white noise in the background. In both cases it was shown that WT was successful in eliminating the noise. However, each case required its unique treatment due to nature of the noise being different. For the second case, the simplest universal soft thresholding technique under $\mathrm{db} 4$ of level 3 was sufficient to get adequate results. At the same time, this method did not work well for the first case. We found that db8 of level 3 with adaptive thresholding gave the best results in reducing keyboard strokes sound in the background.

\section{References}

Aggarwal, R., Rathore, S., Singh, J. K., Tiwari, M., India, M. P., Gupta, V. K., and Khare, A., 2011. Noise Reduction of Speech Signal using Wavelet Transform with Modified Universal Threshold. International Journal of Computer Applications, 20(5), 975-8887.

Bouman, C. A., 2013. Continuous Time Fourier Transform ( CTFT ). Digital Image Processing, 1-5.

Cengiz, Y., Doç, Y., and Arı̈z, U., 2016. An Application for Speech Denoising Using Discrete Wavelet Transform. Biomedical Engineering Meeting, 1-4. 10.1109/BIYOMUT.2016.7849377

Federico, A., and Kaufmann, G. H., 2009. Wavelet Transform, 34(15), 2336-2338. 
Guo, X., Li, Y., Suo, T., and Liang, J., 2017. De-noising of digital image correlation based on stationary wavelet transform. Optics and Lasers in Engineering, 90(July 2016), 161-172. https://doi.org/10.1016/j.optlaseng.2016.10.015

Hazas, M., and Hall, H., 1999. Processing of Non-Stationary Audio Signals. Science, (August).

Huang, W., and Macfarlane, D. L., 2012. Fast Fourier Transform and MATLAB Implementation, 1-26.

Liu, C.-L., 2010. A Tutorial of the Wavelet Transform. National Taiwan University, Department of Electrical Engineering (NTUEE), Taiwan, 172. https://doi.org/10.1111/j.1600-0404.1995.tb01711.x

Misiti, M., Misiti, Y., Oppenheim, G., and Poggi, J.-M., 2009. Wavelet Toolbox TM 4 User' s Guide. The MathWorks Inc., ..., 11-47. Retrieved from http://feihu.eng.ua.edu/NSF_TUES/w7_1a.pdf
Osgood, B., 2007. Lecture Notes for EE 261 The Fourier Transform and its Applications. Stanford University, 428.

Patil, R., 2015. Noise Reduction using Wavelet Transform and Singular Vector Decomposition. Procedia Computer Science, 54, 849-853. https://doi.org/10.1016/j.procs.2015.06.099

Patil, S. S., and Pawar, M. K., 2012. Quality advancement of EEG by wavelet denoising for biomedical analysis. Proceedings - 2012 International Conference on Communication, Information and Computing Technology, ICCICT 2012, 1-6. https://doi.org/10.1109/ICCICT.2012.6398151

Polikar, R. 1994. The Wavelet Tutorial. Internet Resources, 1-67. https://doi.org/10.1088/1751-8113/44/8/085201

Yadav, T. 2016. Denoising and SNR Improvement of ECG Signals Using Wavelet Based Techniques, (October), 678-682. 